\title{
Magnetic Bubbles and Extramixing in Stars
}

\author{
Gerald J. Wasserburg ${ }^{a}$ and Maurizio Busso ${ }^{b}$ \\ ${ }^{a}$ The Lumatic Asylum, Divisionof Geological and Planetary Sciences, California Institute of \\ Technology, Pasadena, CA 91125, USA, gjw@gps.caltech.edu \\ ${ }^{b}$ Department of Physics, University of Perugia, via A. Pascoli, I-06123 Perugia, Italy \\ busso@fisica.unipg.it
}

\begin{abstract}
The possible role of magnetic flux tubes in transporting matter from near the $\mathrm{H}$ shell through the radiative zone and into the convective envelope is explored. It is shown that the required rates of mass transport necessary to provide nuclear processed material to the envelope can be achieved if large magnetic fields are present just above the $\mathrm{H}$ shell in AGB and RGB stars. The required fields in this zone reach $5 \mathrm{MG}$ for the AGB case and 0.5-0.05 MG for the RGB case. It may thus be possible that magnetic bouyancy play a major role in providing the extra mixing needed for these stars.
\end{abstract}

Keywords: Stars: Red Giants; Stars: Evolution; Extended Mixing; MHD.

PACS: 97.20.Li, 97.30.Hk, 97.10.Cv, 95.30Qd

\section{INTRODUCTION}

It has been widely recognized that, during Red Giant Branch (RGB) and Asymptotic Giant Branch (AGB) phases, low mass stars ( 1-3 $\left.M_{\odot}\right)$ must have a weak transport mechanism that moves matter from the vicinity of the $\mathrm{H}$ burning shell, through the stable, non-convecting radiative zone, and injects it into the massive, overlying convective envelope. Evidence for this comes, e.g., from the following observations: 1) The ${ }^{12} \mathrm{C} /{ }^{13} \mathrm{C}$ ratios observed in such stars are lower than predicted by purely convective dredge up; 2 ) High enrichments of $\mathrm{Li}$ (which is usually destroyed in stellar interiors) are sometimes observed and this requires nuclear processing at depth and rapid transport into the envelope; 3) Anomalous abundances of the ${ }^{18} \mathrm{O}$ and ${ }^{17} \mathrm{O}$ isotopes, plus high abundances of ${ }^{26} \mathrm{Al}$ are present in grains of presolar, circumstellar condensates recovered from meteorites. These grains are predominantly from AGB and RGB stars (see Fig. 1) and require some "extra nuclear" processing that is not the result of standard stellar models and is not understood from well defined, dynamical calculations, but requires long-lasting circulation of partially processed material in the radiative layers below the envelope. For reviews of these topics see [1,2].

The amounts of material that need to be transferred are large, being $\sim 1 M_{\odot}$ over the appropriate stellar time scale (RGB or AGB), but with low circulation rates. This process must not disturb the stellar structure or the basic thermonuclear sources.

A variety of mechanisms have been brought forward to address some or all of the above issues: cf. [3,4,5]. Most of these suggestions require some form of ad hoc parameterization. A more recent study [6], using a detailed model of a low mass star,

\footnotetext{
CP1001, $9^{\text {th }}$ Torino Workshop on Evolution and Nucleosynthesis in AGB Stars and the $2^{\text {nd }}$ Perugia Workshop on Nuclear Astrophysics, edited by R. Guandalini, S. Palmerini, and M. Busso (C) 2008 American Institute of Physics 978-0-7354-0520-2/08/\$23.00
} 
showed that, at the boundary of the $\mathrm{H}$ burning shell, an instability is naturally produced due to the combustion of ${ }^{3} \mathrm{He}$.
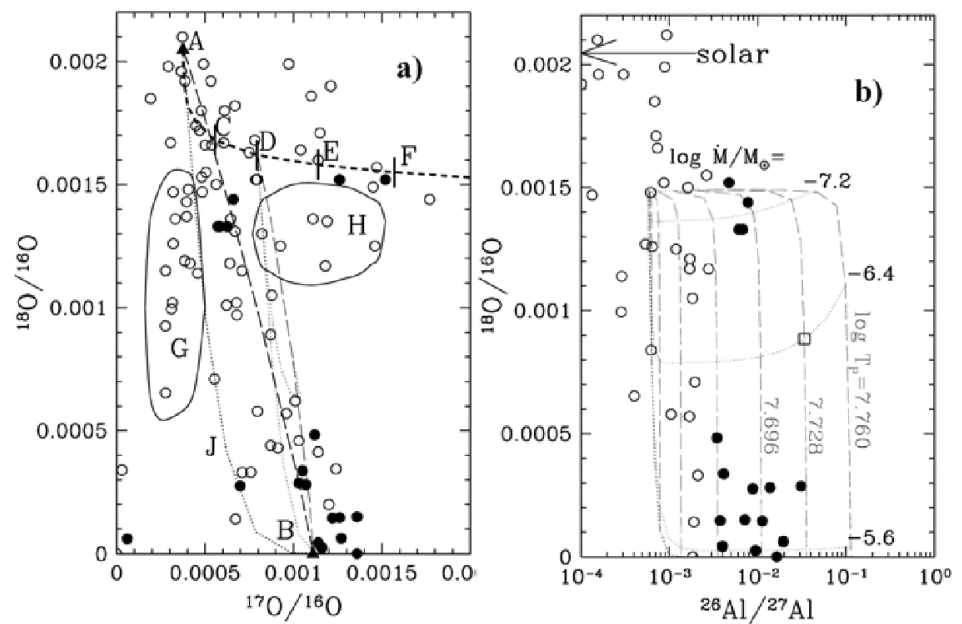

FIGURE 1. a) Diagram, showing the evolution of the oxygen isotopes starting with solar abundances for stars ranging in mass from 1-3 $M_{\odot}$, for 1 st dredge up (trajectory A, C, D, E and F). It is not much changed by 2 nd dredge up. High precision data on circumstellar dust grains show that many of them lie far outside of the possible range for evolutionary models without CBP. Note: point $B$ is the equilibrium value in the $\mathrm{H}$ burning shell where almost complete destruction of ${ }^{18} \mathrm{O}$ occurs (see [7] and references therein). These results unambiguously demonstrate the need for added nuclear processing near the $\mathrm{H}$ shell and injection into the convective envelope. b) The ${ }^{18} \mathrm{O} /{ }^{16} \mathrm{O}-{ }^{26} \mathrm{Al} /{ }^{27} \mathrm{Al}$ diagram, showing the observed grains and the trajectories for a stellar envelope with CBP as a function of $\dot{M}$ and $T_{P}$. Note that, after a drop of ${ }^{18} \mathrm{O} /{ }^{16} \mathrm{O}$ from a Solar ratio to $\sim 0.015$, due to 1 st dredge up, the curves for a fixed $\dot{M}$ are rather flat and not a steep function of temperature. In contrast, the ${ }^{26} \mathrm{Al} /{ }^{27} \mathrm{Al}$ is strongly dependent on $T_{P}$ and requires that it be close to the temperature of the $\mathrm{H}$ burning shell. Thus the ${ }^{18} \mathrm{O} /{ }^{16} \mathrm{O}$ is mostly a measure of $\dot{M}$, and ${ }^{26} \mathrm{Al} /{ }^{27} \mathrm{Al}$ is a thermometer.

This nuclear process causes a molecular weight inversion, resulting in matter transport into the radiative zone, and decreases the abundance of ${ }^{3} \mathrm{He}$ in the convective envelope. This has been recognized as a form of thermohaline mixing [8], especially relevant in stars of about one solar mass. As the efficiency of this mechanism is reduced for increasing mass, it cannot apparently account for the observations outlined above, which concern the most common AGB stars in the galactic disc, whose masses are in the range 1.5 to $2.5 M_{\odot}[9]$.

\section{IS EXTRAMIXING DRIVEN BY MAGNETIC BUOYANCY?}

Investigation of possible stellar stages including 1st and 2nd dredge up showed that many grains lie in a region in the ${ }^{18} \mathrm{O} /{ }^{16} \mathrm{O}-{ }^{17} \mathrm{O} /{ }^{16} \mathrm{O}$ diagram that is forbidden by either normal AGB or RGB evolution (or from Hot Bottom Burning, hereafter HBB) and thus requires additional nuclear processing: such a processing was called "Cool Bottom Processing", or CBP [10]. This term was invented by GJW, on his patio in Pasadena, at the request of Juliana Sackman-Christy, who strongly felt that a name 
was required: it distinguishes between ongoing small levels of nuclear processing from efficient envelope burning (HBB). $\mathrm{CBP}$ is amongst the phenomenological transport models under consideration to explain the extra nuclear burning. This model has been extensively explored by [11] and by [7]. It was shown that CBP may be treated in a simple "Box Car" model, in which matter circulates from the base of the convective envelope to a depth where the temperature is $T_{P}$ and then moves upward into the envelope. This produces a small amount of nuclear processing at each pass and only depends on the path integral of the nuclear reaction rates and the mass flow rate. The processing does not significantly disturb the overall stellar structure.

The only parameters that define the results are the mass flow rate $(\dot{M})$ and the maximum temperature $T_{P}$ reached (for a star of a given mass and metallicity). The schematic Box Car flow pattern was simply assumed and no mechanism was proposed to drive the flow. The results of this approach are that the observed range of isotopic shifts can be very plausibly explained by $\mathrm{CBP}$ and that its conditions $\dot{M}$ and $T_{P}$ can be easily defined. These results permit a clear interpretation of most observational data. However, the question remains as to the physical mechanism that causes the transport and what the velocity field must be. In this report, we will present the possibility that buoyancy of magnetic bubbles might be the cause of the transport. E. N. Parker first proposed [12] that "magnetic buoyancy" might be a cause of Sunspots. Gurm and Wentzel then studied the conditions of convection in a zone of radiative equilibrium due to B fields [13]. In a very insightful paper, Hubbard and Dearborn proposed [14] that magnetic mixing might be a mechanism to explain the ${ }^{12} \mathrm{C} /{ }^{13} \mathrm{C}$ data and would have direct effects on ${ }^{18} \mathrm{O},{ }^{17} \mathrm{O}$ and $\mathrm{C} / \mathrm{N}$ (there was no data available at that time).

In 2005, the senior author of this paper received an inquiry from Maurizio Busso, who said "What do you think about magnetic bubbles?" (see Fig. 2). He responded, "That is a great idea, why don't you do it". After some time and with considerable work with Andrea Calandra, Maurizio, upon visiting with the Wasserburg family in Florence (Oregon), showed the results of their preliminary work and asked GJW if he would join them. GJW responded that he had little knowledge of the subject as the last intimate contact that he had with magneto-hydrodynamics (MHD) was when he took Chandrasekhar's course more than 56 years ago. This did not dissuade Maurizio and it was agreed to make a joint effort. The idea was that if material were magnetized near the $\mathrm{H}$ burning shell, then the magnetic pressure $\left(B^{2} / 8 \pi\right)$ might produce sufficient buoyancy to transport matter up to and into the convective zone. This subject had been already considered by many authors in various scenarios, after the early suggestions by E. N. Parker for the case of the Sun [15]. In particular, the interested reader is referred to [16]. McDonald and Mullan studied shear instability in rotating RGB stars [5] and showed that it could result in B fields between the $\mathrm{H}$ shell and the convective envelope.

The fundamental issues were: 1) the basic MHD mechanisms that must provide the fields; 2) the consequences that would follow if magnetic bubbles were to transport sufficient material to address the circulation that $\mathrm{CBP}$ required. After consideration of the great complexity associated with the MHD problem, it was chosen to ignore the first basic question (n. 1 above) and focus on problem number 2 . We now have the special privilege to give the first public presentation of this work [17], that is just now 
published (here it will be referred to as BWNC). The senior author guesses that this is a birthday present for him.

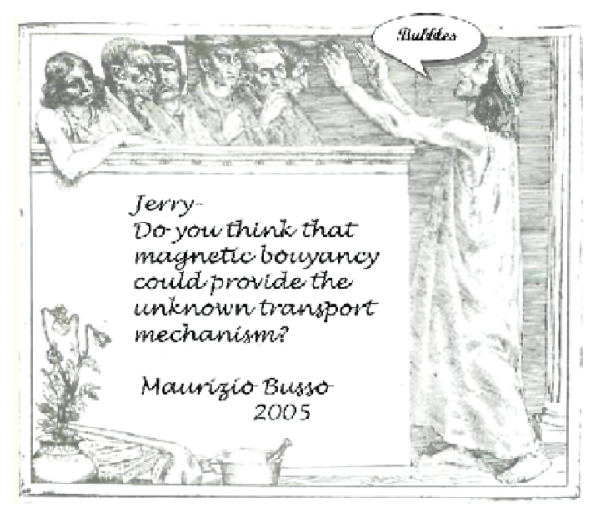

FIGURE 2. A message from MB to GJW in 2005.

\section{A PHENOMENOLOGICAL MODEL FOR MAGNETIC CBP}

In the matter at hand, the question is: "What do we know and how far can it take us?". We know the stellar structure of low mass stars from extensive and intensive investigations. Hence, we know the density, pressure, composition and structure of stars over their evolution with a high degree of certainty. Using this basis, as provided by several of you in the audience, we can determine, using the observational data and the CPB model, the rate of mass transfer $(\dot{M})$ and the temperature $T_{P}$ (or depth) to which the circulation must reach. This is all that is available to us now.

We show the data for a star of initial mass $1.5 M_{\odot}$ during the RGB phase, taking into account CBP (see Fig. 3). It can be seen that to achieve the required value of ${ }^{12} \mathrm{C} /$ ${ }^{13} \mathrm{C} \sim 10-12$ over the time spent on the RGB, this would require $\dot{M}$ between $4 \times 10^{-7}$ and $4 \times 10^{-8} M_{\odot} / \mathrm{yr}$. This result is not very dependent on the temperature $T_{P}$ (values of $\log$ $T_{P} / T_{H} \cong-0.2$ to -0.25 are sufficient).

For the AGB phase it can be seen from Fig. 1 that many observed data points require major depletion of ${ }^{18} \mathrm{O}$. As seen in Fig. $1 \mathrm{~b}$ ), the ${ }^{18} \mathrm{O} /{ }^{16} \mathrm{O}$ versus ${ }^{26} \mathrm{Al} /{ }^{27} \mathrm{Al}$ trajectories of CBP as a function of $\dot{M}$ and $T_{P}$ show that ${ }^{26} \mathrm{Al} /{ }^{27} \mathrm{Al}$ is essentially a thermometer, while ${ }^{18} \mathrm{O} /{ }^{16} \mathrm{O}$ is most strongly dependent on $\dot{M}$. From an analysis of the CBP results [7] one derives that, in order to account for the general effects on the AGB, we require $\dot{M}$ values between $4 \times 10^{-7}$ and $2.5 \times 10^{-6} M_{\odot} / \mathrm{yr}$ and temperature values such that $\log T_{P} / T_{H} \cong-0.1$.

From the above considerations, we therefore have clear guides to the values of $\dot{M}$ and $T_{P}$ required for a magnetic bubble transport model. As typical rates, we take from the above considerations $\dot{M}=10^{-6}$ and $4 \times 10^{-8} M_{\odot} / \mathrm{yr}$ for the AGB and RGB, respectively. 
We shall consider, as a reference, the case of a star of initial mass $1.5 M_{\odot}$ with a metallicity half of the Solar value and an internal structure at the RGB and AGB phases as computed previously $[18,19]$, including mass loss with the parameterization presented in [20].

\begin{tabular}{lcc} 
TABLE 1. & & \\
\hline & AGB & RGB \\
\hline$\dot{M}(M \odot / \mathrm{yr})$ & $10^{-6}$ & $4 \times 10^{-8}$ \\
$f_{M T} x f_{v s}$ & $2 \times 10^{-7}$ & $8.7 \times 10^{-10}$ \\
$v_{T R A N S}$ & $1 \mathrm{~m} / \mathrm{s}$ & $1 \mathrm{~m} / \mathrm{s}$ \\
$f_{M T}$ & $7 \times 10^{-2}$ & $2 \times 10^{-4}$ \\
\hline
\end{tabular}

The first issue is whether the transport across the boundary between the upper part of the radiative zone and the base of the convective layer will cause any difficulties. If VTRANS is the average velocity of transport across this interface and $f_{M T}$ the fraction of the mass at the interface that came from CBP, then the relationship with $M$ is:

$$
\dot{M}=\mathrm{f}_{\mathrm{MT}} \mathrm{v}_{\mathrm{TRANS}} \mathrm{x} 4 \pi \mathrm{R}^{2} \rho=\mathrm{f}_{\mathrm{MT}} \mathrm{f}_{\mathrm{VS}} \mathrm{v}_{\mathrm{SOUND}} \mathrm{x} 4 \pi \mathrm{R}^{2} \rho
$$

where vSOUND is the sound speed, and both $f_{M T}$ and $f_{V S}$ must be $<<1$ (see again BWNC for details). The results for the product $\left(f_{M T} \times f_{V S}\right)$ are shown in Table 1 for

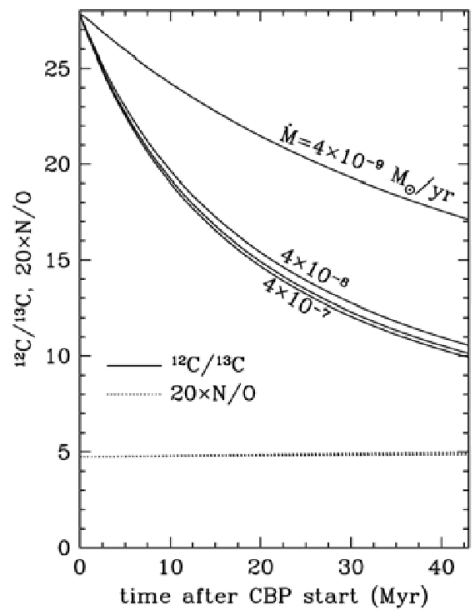

FIGURE 3. Evolution of the ${ }^{12} \mathrm{C} /{ }^{13} \mathrm{C}$ ratio in the envelope of a $1.5 M_{\odot}$ star during the RGB phase. Evolution curves for different values of $\dot{M}$ are shown. To achieve ${ }^{12} \mathrm{C} /{ }^{13} \mathrm{C} \sim 10$ at the end of the RGB phase, the requirements on $\dot{M}$ are shown (see BWNC).

both cases. If we assume that $\mathrm{v}_{\text {TRANS }} \sim 1 \mathrm{~m} / \mathrm{s}$, as is inferred for the Solar case, then values of $f_{M T}$ can be obtained. It is clear for the RGB case that CBP only requires a 
very small part of the matter at the upper interface to be from the extra transport. For the case of the AGB, it is small but substantial. As we will show later, the velocity of the ascending magnetic bubbles on the AGB are much more rapid $(1 \mathrm{~km} / \mathrm{s})$ and it is reasonable to consider that they will readily intrude into the convective layer at speeds much greater than $1 \mathrm{~m} / \mathrm{s}$. We therefore do not consider that the flow across this upper boundary be a problem.

We also note that the frequency with which the magnetic bubbles must cross the upper boundary is: $\dot{M} / M_{\text {bubble }}=\hat{V} / \Delta R$. Here $M_{\text {bubble }}$ is the mass of the bubble, $\hat{V}$ is its average velocity over the trajectory and $\Delta R$ is the distance traveled. Note also that, if only one "event" occurs per month, then this would require a bubble of $\sim 1000 \mathrm{~km}$ in scale for the RGB case.

Now we turn to the matter of the transport from the processing zone through the radiative zone. This requires that we define the geometry of the "bubble" and the dynamics of motion. We assume here that the bubble is a torus, - a toroidal magnetic flux tube (Fig. 4a) and is transported radially from the CBP processing zone (temperature $T_{P}$ ) to the base of the convective envelope with a velocity $v(R)$ (see Fig. 4b). The expression for the average velocity is given in the figure. The equation relating the density shift in the magnetized material of the torus to the magnetic field is $\left(\rho-\rho^{\prime}\right) / \rho(R)=B^{2} / 8 \pi P(R)$. The buoyant force per unit length on the torus is: $\mathrm{p} a^{2}(R) g(R)\left(\mathrm{r}-\mathrm{r}^{\prime}\right)$, where $a(R)$ is the cross sectional radius of the torus and $g(R)$ is the local acceleration of gravity. The drag force per unit length on the torus is $1 / 2 C_{D R A G} \rho a(R) v^{2}(r)$. We assume mass conservation and magnetic flux conservation for the flux tube giving: $R a^{2}(R) \rho(R)=$ const. and $B(R) a^{2}(R)=$ const. The mass of the toroidal flux tube is $2 \pi^{2} \rho_{0} R_{0} a_{0}{ }^{2}$.
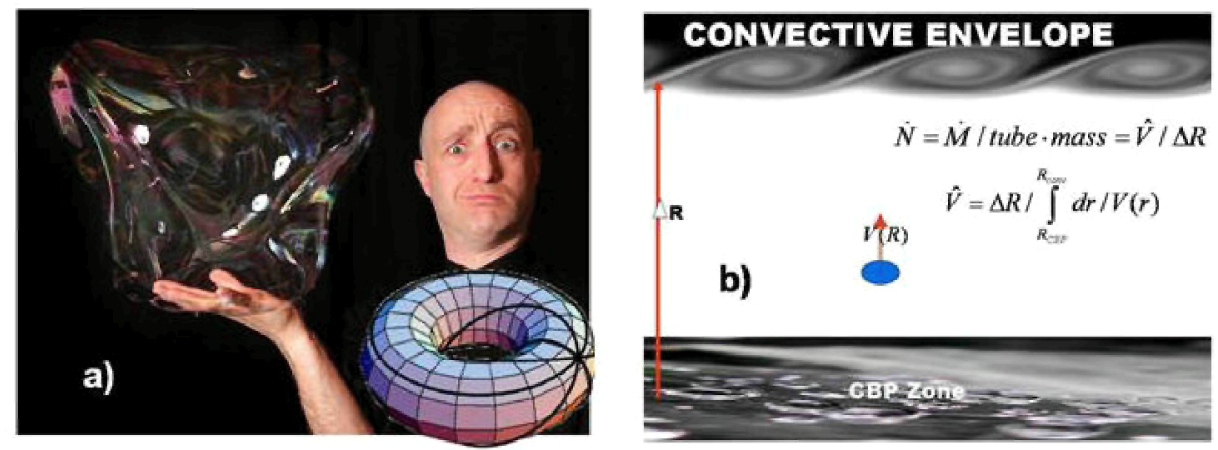

FIGURE 4. a) The problem of bubble geometry. A simple torus was chosen representing an undeformed magnetic flux tube; b) Cartoon showing the transport between the CPB processing region and the convective envelope .

This treatment follows original works on flux tubes in the Sun $[15,21]$. Using these relationships, we obtain for the velocity as a function of radius:

$$
v(r)=1 / 2\left(\rho(r) / \rho_{0}\right)^{3 / 4}\left(r / r_{0}\right)^{-1 / 4}\left(g_{0} a_{0} / C_{D}\right)^{1 / 2}\left\{B_{0} / \sqrt{P(r)}\right\}
$$


The values with subscript 0 correspond to the deepest processing zone $\left(T_{P}\right)$. The drag coefficient $C_{D}$ is typically estimated at 1 , but much lower values, down to 0.04 are possible. Note that $C_{D}$ occurs as $B d \sqrt{C_{D}}$, thus even this wide uncertainly range does not grossly affect $B_{0}$. We have taken $g(R) / g_{0}=\left(R_{0} R\right)^{2}$ as there is very little range in mass across the radiative zone. In calculating $v(R)$ we have used the values of $\rho$ and $P$ determined in the stellar model. We have inferred the values of $a_{0}$ from Solar observations. The resulting curves for $v(R)$ and $\hat{v}$ are shown in Fig. 5. Table 2 shows the required magnetic fields $\left(B_{0}\right)$ at the processing zone, the initial velocities and average velocities to obtain the required flows for each case. The value of $B_{C O N V}$ at the base of the convective envelope is also given. The frequencies for a bubble event for the parameters used are $\dot{N}=2.6 \times 10^{-6} / \mathrm{s}$ (7 per month) for the AGB, and $\dot{N}=1.6 \times 10^{-7} / \mathrm{s}$ (5 per year) for the RGB.

TABLE 2.

\begin{tabular}{lccc}
\hline & AGB & RGB I & RGB II \\
\hline$a_{0}(\mathrm{~km})$ & 65 & 65 & 150 \\
$\hat{v}(\mathrm{~km} / \mathrm{s})$ & 1.45 & 0.098 & 0.018 \\
$v_{o}(\mathrm{~km} / \mathrm{s})$ & 6.8 & 0.336 & 0.062 \\
$B_{0}(G)$ & $5 \times 10^{6}$ & $3.8 \times 10^{5}$ & $4.8 \times 10^{4}$ \\
$B_{C O N V}(G)$ & $8.6 \times 10^{3}$ & $3.4 \times 10^{3}$ & $4.4 \times 10^{2}$ \\
\hline
\end{tabular}

From the results given in Table 2, it can be seen that:

1) For the RGB case the fields at the processing zone are substantial, but are much lower at the base of the convective envelope;

2) For the AGB case the required fields at the processing zone are quite large ( $\sim 5$ Megagauss), but again they are much reduced at the base of the envelope.

3) The size of the hypothecated flux tubes is justifiable in terms of the Solar observations.

4) The velocity of upward transport by the bubbles is quite rapid so that very little nuclear processing will result from this part of the trajectory.

5) The hypothecated velocity of trajectory in most of the treatments $[7,11]$ assumed a rather low velocity field $(\cong 1 \mathrm{~m} / \mathrm{s})$ for both downward and upward motion. Thus, in order to accomplish the nuclear processing, the magnetic bubble model requires that the complimentary downward flow (D) be broad and slow $\left(f_{D} \cong 0.5\right.$, as in WBS). 


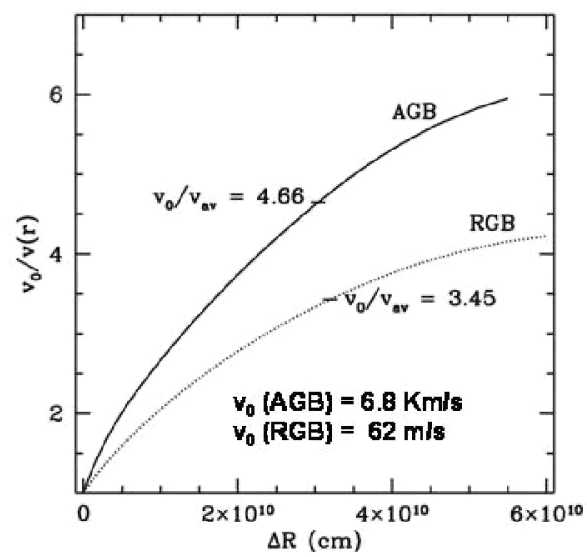

FIGURE 5. Curves showing the velocities of ascent as a function of distance for the AGB and RGB cases (relative to the initial velocities at $T_{P}$ ).

We must emphasize that the model outlined here was guided by the results from SOHO using Helioseismology, which proved the differential rotation of the subenvelope zones of the Sun relative to the rather rigid interior. This motion penetrates to a depth $\sim 0.04 R \odot$ and $0.01 M \odot$ thick into the radiative zone. This results in a weakly convective velocity field that penetrates into the radiative zone immediately below the envelope, a region called the Tachocline. It is further inferred that the fields deep in the radiative tachocline must exceed $\sim 10^{5}$ Gauss $[22,23]$. For a thorough presentation of the structure and flow dynamics of the Sun the reader is referred to the site: http://solarphysics.livingreviews.org/About/copyright.html, which contains an excellent review [24].

The fundamental issue of the basic MHD mechanisms that must provide these observed fields remains to be resolved and is the subject of intense investigation in the Solar MHD community. We have simply assumed that they also exist for RGB and AGB stars.

There is then the issue of whether the available observations on RGB or AGB stars are in conflict with the proposed model. There is no direct observational evidence of strong magnetic fields in either RGB or AGB stars. A rough estimate for AGB stars, using flux conservation extrapolated to the surface, gives surface fields of up to 20G, in line with values inferred (from observational estimates in the circumstellar envelope) by [25]. This is compatible with the fact that AGB stars are not observed to have X-ray emitting coronae. For the RGB case the surface fields would again be small, in the range from $\sim 1$ to a few G. However, it is quite plausible that the fields we find at the base of the envelope would be greatly subdued, far below the values assuming flux conservation, when the flux tubes are engulfed in the very massive overlying convecting stellar envelope. 


\section{DISCUSSION AND PRELIMINARY CONCLUSIONS}

In this note we have reported on the possibility that magnetic buoyancy be responsible for driving extramixing processes in red giants, during their first and second ascent on the giant branch. In our original work, published in the Astrophysical Journal, several important earlier papers were overlooked. We here have noted and recognized these critical contributions.

With regard to the high fields found to be necessary for the AGB case, we note that plasma jets of magnetic origin have been observed for many planetary nebulae [26]. A planetary nebula is the result of the end of AGB evolution, when the envelope is blown off, leaving a white dwarf remnant. Blackman et al. considered the development of dynamos in AGB stars as the origin of magnetic fields shaping planetary nebulae [27]. In their calculations they inferred fields of $5 \times 10^{4} \mathrm{G}$ at the base of the AGB convective envelope (at a radius of $1.5 R_{\odot}$ ) in order to explain the collimation. $B$ values of up to a few $\times 10^{6} \mathrm{G}$ were inferred in the case of low filling factors (which is in fact our case, due to the low values of $f_{M T}$ ). There is some problem in sustaining such fields [28]. In general, there is some independent evidence for significant internal magnetic fields in AGB stars. Magnetic fields in planetary nebulae are considered to be the cause of the bi-polar outflows and are the subject of intense investigation. In particular Nordhaus and Blackman are seeking possible scenarios that might provide the ongoing (or frequently recurring) high fields as presented here -- see their report in this volume [29].

It is further of note that white dwarfs, which are the end product of AGB evolution, sometimes show very high fields. Recent studies have revived the old hypothesis [30] that these fields might be fossil remnants of the stellar ones; for modern studies on this subject see $[31,32,33]$. About $10-20 \%$ of the available white dwarf sample show fields larger than $1 \mathrm{MG}$ (see Fig. 6), and up to 2 Gigagauss [34]. These observations suggest that possibly, the high fields of such white dwarfs might be inherited from the AGB precursors, with high enhancement factors ensuing upon the expulsion of the envelope.

From the arguments presented in this report we conclude that magnetic buoyancy is a very plausible mechanism for transporting material upward from near the $H$ shell into the convective envelope, for both RGB and AGB stars. This requires very high internal fields for those AGB stars with extensive CBP. We have only given phenomenological arguments regarding the mass transport, assuming that the stars provide the required magnetic fields. The fundamental issue as to how the fields are generated by current flows is not addressed in our work.

Gene Parker, in commenting on this work stated "I feel that your paper makes a fundamental point, that deep mixing is an essential part of the evolution on the RGB and the AGB. What causes the convection? I understand from your paper that the only theoretical possibility that cannot be ruled out at the present time is the magnetic buoyancy of Megagauss fields. It is not possible, so far as I am aware, to explain the presence of a Million Gauss in the radiative zone, or anywhere else. We should recognize that a similar problem exists in the Sun. [....] Nowhere is there enough convective muscle to manipulate so strong a field. So one turns to schemes whereby a 
weaker field, of say, 5 KiloGauss, is greatly intensified by the upward bulging $\Omega-$ shaped loops that exist at intervals along the flux bundle. The upward convection, when an $\Omega$-loop is formed, sucks the gas out of the portion of the flux bundle that remains deep down in the convective zone. [...] If there is some hope, you can find it written up in [21]." An analysis of the magnetic instabilities suitable to enhance otherwise weaker fields was later presented in [34].

It is our hope that future investigations will be found to provide the "convective muscle" required by many stars, including the Sun, to yield the apparently necessary magnetic fields.

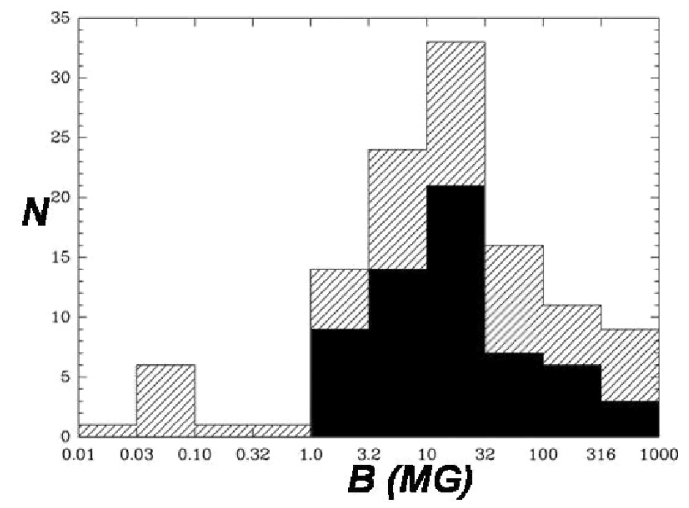

FIGURE 6. Histogram of magnetic white dwarfs in equal intervals of $\log B(\mathrm{MG})$. SDSS discoveries are shaded black; the distribution for all known magnetic white dwarfs is hatched [34].

\section{ACKNOWLEDGEMENTS}

GJW acknowledges the support of DOE-FG03-88ER13851, the generosity of the Epsilon foundation, and Caltech's contribution 9003(1124). M.B. thanks support from the Italian Ministry of research, through the contract PRIN2006-022731.

\section{REFERENCES}

1. C. Charbonnel, In "Origin and Evolution of the Elements", ed. A. McWilliam and M. Rauch, (Pasadena: Carnegie Observatories), p. 59, (2004)

2. G.J. Wasserburg, M. Busso, R. Gallino, and K.M. Nollett, Nuc.Phys. A 777, 5 (2006)

3. C. Charbonnel and J.D.Jr. Do Nascimiento, $A \& A$ 336, 915 (1998)

4. P.A. Denissenkov, G.S. Da Costa, J.E. Norris, and A. Weiss, $A \& A$ 333, 926 (1998)

5. J. McDonald and D.J. Mullan, $A p J$ 598, 560 (2003)

6. P.P. Eggleton, D.S.P. Dearborn, and J.C. Lattanzio, Science 314, 1580 (2006) 
7. K.M. Nollett, M. Busso, and G.J. Wasserburg, $A p J$ 582, 1036 (2003)

8. C. Charbonnel and J.P. Zahn, $A \& A$ 467, L15 (2007)

9. M. Cantiello, H. Hoekstra, N. Langer, and A.J.T. Poelarends in "Unsolved Problems in Stellar Physics", AIP Conf. Proc. 948, p. 73 (2007)

10. A.I. Boothrpyd, I.-J. Sackman, and G.J. Wasserburg, $A p J$ 430, L77 (1995)

11. G.J. Wasserburg, A.I. Boothroyd, and I.-J. Sackmann, ApJ 447, L37 (1995)

12. E.N. Parker, $A p J$ 121, 491 (1955)

13. H.S. Gurm and D.G. Wentzel, $A p J$ 149, 139 (1967)

14. E.N. Hubbard and D.S.P. Dearborn, $A p J$ 239, 248 (1980)

15. E.N. Parker, Ap. Space Sci. 31, 261(1974)

16. H.C. Spruit, $A \& A$ 381, 923 (2002)

17. M. Busso, G.J. Wasserburg, K.M. Nollett, and A. Calandra, ApJ 671, 802 (2007)

18. O. Straniero, A. Chieffi, M. Limongi, et al., ApJ 478, 332 (1997)

19. M. Busso, R. Gallino, and. G.J. Wasserburg, PASA 20, 356 (2003)

20. D. Reimers, in "Problems in Stellar Atmospheres and Envelopes". Ed. B. Bascheck, W.H. Kegel, G. Traving (New York: Springer-Verlag Inc.), p. 229 (1975)

21. E.N. Parker, $A p J$ 433, 867 (1994)

22. M. Rempel, M. Schuessler, and G. Toth, $A \& A$ 363, 789 (2000)

23. M. Schuessler and M. Rempel, in "From Solar Min to Max: Half a Solar Cycle with SOHO", ed. A. Wilson, ESA SP-508 (Nordwjik:ESA Publ. Division), p. 499 (2002)

24. M.S. Miesch, Living Rev. Solar Phys. 2, 1 (2005)

25. F. Herpin, A. Baudry, C. Thum, et al., $A \& A$ 450, 667 (2006)

26. J.H. Kastner, B. Balick, E.G. Blackman, et al., ApJ 591, L37 (2003)

27. E.G. Blackman, A. Frank, J.A. Markiel, et al., Nature 409, 485 (2001)

28. J. Nordhaus, E.G. Blackman, and A. Franck, MNRAS 376, 599 (2007)

29. J. Nordhaus and E.G. Blackman, this volume (2007)

30. E.N. Hubbard and D.S.P. Dearborn, $A p J$ 247, 236 (1981)

31. C.A. Tout, D.T. Wickramasinge, and L. Ferrario, MNRAS 355, L13 (2004)

32. D.T. Wickramasinge and L. Ferrario, MNRAS 356, 1576 (2005)

33. G. Valyavin, S. Bagnulo, S. Fabrika, et al., ApJ 648, 559 (2006)

34. G.D. Schmidt, H.C. Harris, J. Liebert, et al., $A p J$ 595, 1101 (2003)

35. H.C. Spruit, $A \& A \quad 349,189$ (1999) 